\title{
MJN EFFECTIVE COMMUNICATION AMONG DOCTORS AND NURSES: BARRIERS AS PERCEIVED BY DOCTORS
}

\author{
Annamma Kunjukunju*, Aini Ahmad \\ School of Nursing, KPJ Healthcare University College, Malaysia \\ *Correspondence Author's Email: annjoe212@gmail.com
}

\begin{abstract}
Background: Hospital medicine is characterized as a multifaceted team-based activity with a necessity for a high level of accuracy, timely, and reliable communication. Effective communication among healthcare providers is the key to successful collaboration within the healthcare system. Effective communication can improve patient safety, job satisfaction, and reduce burn out syndrome among nurses. The purpose of the study was to identify the factors contributing to the competency gap between and doctors in four private hospitals in Malaysia. Method: The study used a descriptive qualitative design to elicit the experience of 15 doctors from three private hospitals. A semi-structured interview was conducted to collect the data. The data were analyzed using Colaizzi's method for thematic data analysis. Results: The findings of the study suggested three main categories as the factors contributing to the communication gap among nurses and doctors as perceived by specialist doctors. The three categories include nurses training attributes, graduate nurse attributes, and work environment attributes. The nurses training attributes include entry requirements for student's recruitment into the nursing program, undergraduate training process, and assessment of student nurses. The graduate nurse's attributes had nine elements. The elements include a post-registration thirst for knowledge, medical English knowledge, self-confidence, stress management skills, critical thinking, and clinical judgment skills, perceived power on the authority of doctors, passion to work, time management skills as well as stress and personal factors of nurses. The working environment attributes included workload of nurses, teamwork among nurses Preceptorship, and appreciation of nurses by the organization. The informants also suggested measures to overcome the competency gaps among nurses. Conclusion: In conclusion, the nurse's communication competency is a vital factor in determining patient safety and quality of care. The quality of nursing care has a significant impact on the image of any health care organization. Therefore, it is crucial to identify the factors contributing to poor communication among nurses and doctors and to initiate interventions to fill the communication gap.
\end{abstract}

\section{Keywords: Registered Nurse, Communication, Interdisciplinary Communication, Nurse-Doctor Communication}

\section{INTRODUCTION}

It is imperative that everyone in the healthcare industry have excellent communication skills to become a good listener and communicator. Nurses and doctors are the closest partners in any healthcare organizations and have a common goal, to provide care. The communication among the doctors and nurses is crucial for the enhancement of teamwork culture and to safeguard patients' safety. Under-appreciation of the importance of effective nurse-doctor skills and inadequate communication skill training can have serious implication in nursing practice (Hsu, Chang \& Hsieh, 2015). The Joint Commission established
International Patient Safety Goals (IPSGs) in the year 2002. The IPSGs were developed to help accredited healthcare organizations to address specific areas of concern regarding patient safety and the number two IPSG was to "Improve Effective Communication" (Sorra et al., 2016).

The relationship between the doctor and the nurse is also an exceptional one. There are few professions where the degree of mutual respect and cooperation between co-workers is as intense as that between the doctor and nurse (Stein, 1967). Doctor's obtain information about patients from varying sources like medical history, physical examination, laboratory 
investigations. However, nurses are with the patients $24 / 7$, and as such, the information provided by nurses regarding patient's progress is the most vital information to make treatment recommendations. Deficient communication among healthcare providers can result in frustration and distrust among healthcare providers and may lead to inferior level of care and a higher risk of error (Burke, Boal \& Mitchell, 2004). The purpose of the study is to identify the factors contributing to poor nurse-doctor communication as perceived by the doctors.

\section{Background of the study}

Effective communication is a hallmark of safe patient care. The study has been conducted in three selected private hospitals in Malaysia, which are under one flagship of one healthcare organization. All the hospitals are tertiary level specialist hospitals providing a wide range of specialist medical services across all medical disciplines. All the registered nurses possess a Diploma in nursing qualification, which is the primary nursing qualification for a Registered nurse in Malaysia. They had been trained mainly within the same Healthcare organization. However, the organization also have a good number of nurses trained in other Schools of Nursing across Malaysia. All the nurses working for the organization and providing direct patient care are locals. The specialist had been trained in varieties of settings across the globe. Nurses and doctors are the closest partners in healthcare delivery. From the researcher's personal experience as a nurse educator, there had been criticisms about junior nurse's communication competencies, especially in specialty units. The purpose of this study is to determine the contributing factors of nurse-doctor communication gaps as perceived by the doctors.

\section{Purpose of the study}

The purpose of the study was to identify the factors which contributes to nurse-doctor communication gaps as perceived by the doctors.

\section{METHODOLOGY}

\section{Research design}

The study used a qualitative method with descriptive explorative design to explore factors affecting the competency development of nurses. The study used an expert interview for data collection. The study used source triangulation by recruiting 15 doctors from three different hospitals and various disciplines for data saturation.

\section{Study settings}

The study settings involved three private hospitals in Malaysia. All the three hospitals are under the management of KPJ Healthcare organization. The medical specialists from various discipline were involved in the study.

\section{Sampling technique}

A purposive sampling technique was used for the study. The expert was personally contacted over the phone by the researchers, and an appointment was made for the interview in their clinics.

\section{Inclusion criteria}

The specialist doctors who had been willing to give the explanatory consent was included as the respondents for the study.

\section{Exclusion criteria}

The specialist doctors who were not willing to participate and sign explanatory statement was excluded for the study.

\section{Data collection method}

The study used semi-structured questions for data collection. The semi-structured interview was conducted at the hospital premises. Before the data collection, the researcher had obtained permission from the Chief Executive Officer (CEO) of the respective hospitals. With the permission and referral of the hospital management, the researcher personally contacted the specialist doctors and introduced themselves, and an appointment was obtained to conduct the semi-structured interview. Before the commencement of the interview, the topic and purpose of the study was reinstated to the respondents and obtained the written consent using the explanatory statement. Then, the researcher collected demographic data's followed by a semi-structured interview which was conducted regarding the competency of graduate nurses. All the interviews were tape recorded with the consent of the respondents.

\section{Ethical clearance}

This study has obtained the Ethical Clearance / approval from the Ethical Committee. An explanatory statement was given, and consent was obtained from the respondents before the data collection. The data in the tape recorder was transferred to the laptop and was password protected to be accessible only to the research team. The transcribed verbatim was stored in a 
dedicated box to protect the confidentiality of the data. The study used semi-structured questions for data collection.

\section{Data analysis method}

The interviews were tape recorded, and verbatim was transcribed. The data analysis used Manual qualitative analysis from the field notes and the verbatim. The transcript was read and re-read independently by the researchers to obtain the themes related to factors affecting the competency gap among junior registered nurses.

\section{RESULTS}

\section{Demographic profile of the respondents}

Table 1: The demographic profile of the respondents of this study

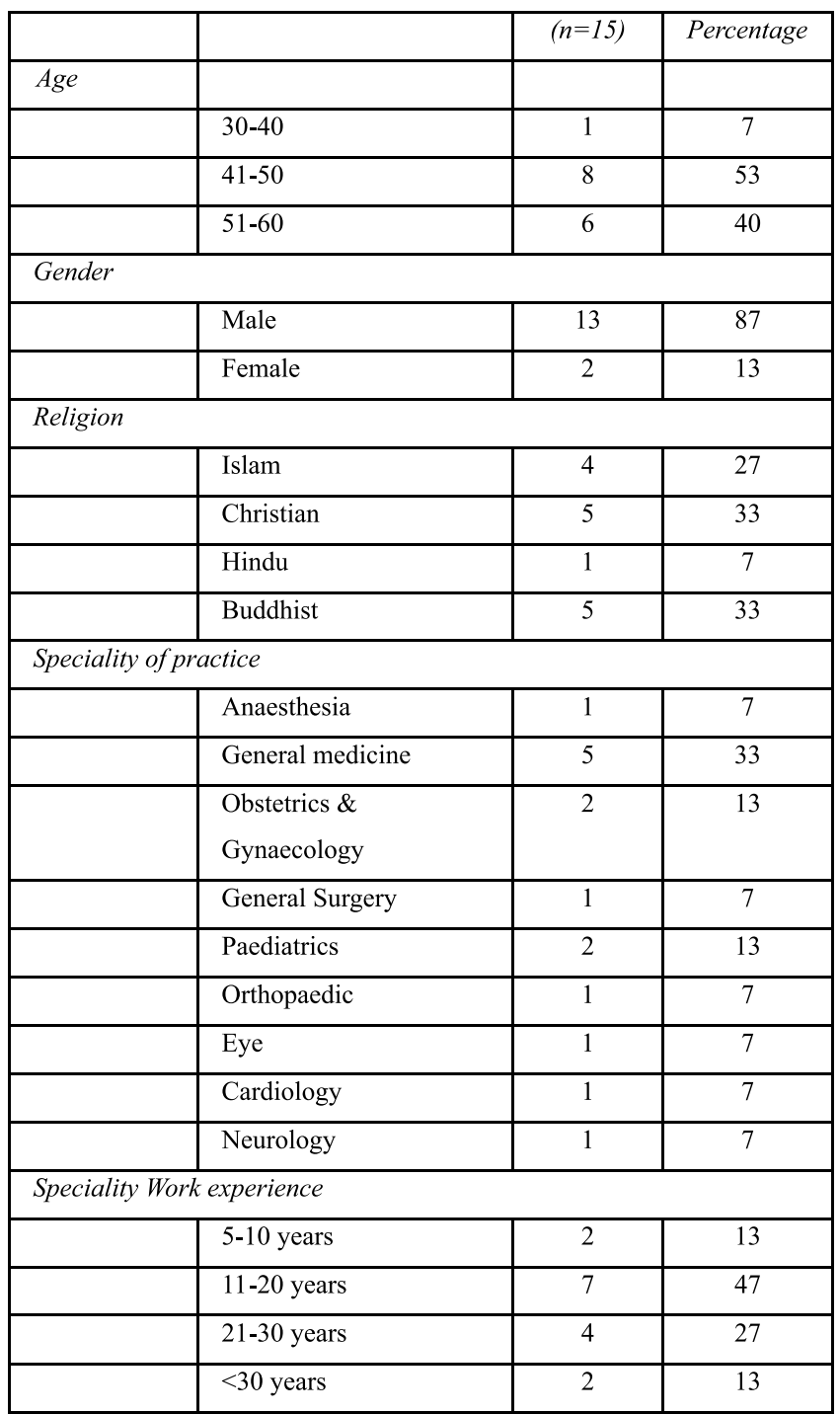

The majority $(53 \%)$ of the respondents were between the age group of 51-60 years of age and were males. Christians and Buddhist had been the main religion of the specialist. The specialist had been from a various specialty with $33 \%$ from General medicine. The majority $(47 \%)$ had more than ten years of clinical practice in the respective medical discipline.

\section{Factors determine communication gap between doctors and nurses.}

The findings of the study revealed three main factors contributing nurse-doctor communication gap. The details are displayed in figure 1.
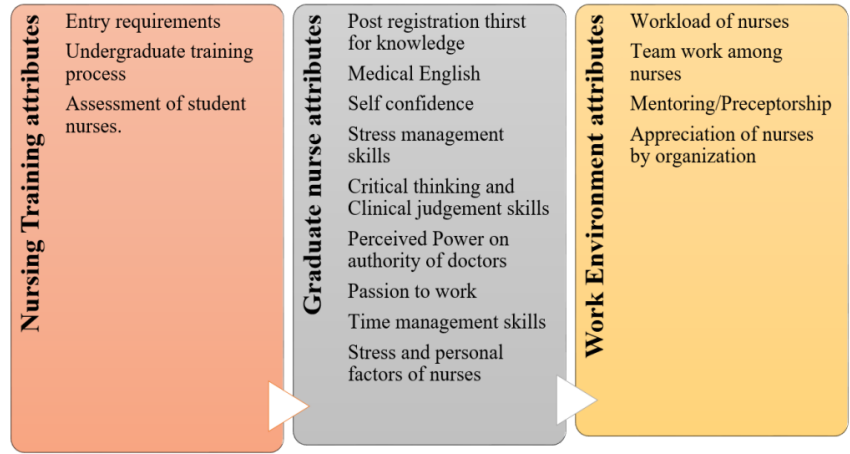

Figure 1: Registered Nurses Competency Determinants

\section{Nursing Training attributes}

\section{Entry requirements:}

Doctors recommended to include aptitude testing as part of the recruitment and selection process of nursing students before admission into the nursing program to ensure that the right candidates are admitted into the nursing program. They highlighted that the nursing profession is not for everyone. Aptitude test will ensure that the candidates possess the right traits to be a successful and caring nurse in the future.

\section{ii. Undergraduate Training process:}

Undergraduate Training needs to be well-structured, especially during clinical practice. The student nurses are too young to behave like professional. In the clinical area, senior nurses should be available to guide the student nurses. However, due to the workload of the senior nurses, they may not be able to find adequate time to guide student nurses. Moreover, student nurses need to be trained in a very controlled clinical set up for more experience with adequate clinical supervision. The nursing students should have enough exposure to a variety of cases to learn varying severity of cases like Dengue fever and others. Academics also must contribute to student clinical learning. 
Conversely, the students need to have a focus to learn from the clinical specialties when they are students. The process of learning should be during their student's life and must not start all over again once they become staff nurses. Students must be committed to learning. During Clinical Training, there are 'some stress' in learning, but nursing students should not take the worth of clinical knowledge for granted. They should be made in charge of a certain number of patients as students. The student nurses need to have close supervision and clinical placement should not be just complete log book requirements.

\section{iii. Assessment of students:}

The assessment in nursing must stress on clinical communication competency development. The assessment during training should be able to identify the communication competency gap among students and take actions to resolve the gaps.

\section{Graduate Nurses attributes}

\section{Post registration thirst for knowledge:}

Knowledge is the critical factor which determines confidence and courage for inter-professional communication. Three years of Diploma in nursing training only prepare a generalist nurse. In order to become a specialists nurse, they must be able to perform in highly specialized areas and match with the expectation of the medical specialists who have vast experience in the medical specialty. Nurses need to focus and have interest in the subspecialty upon graduation. Right attitude to learning is essential for professional nurses. The knowledge they possess will differentiate a nurse who is a professional practitioner from a task orientated worker. There should be a learning process among nurses and not act like passengers or messengers in the ward. Basic knowledge in the specialty is essential. Nurses must believe in lifelong learning. Learning must happen every day among nurses while involved in clinical practice. The thirst for new knowledge should be there in all professional nurses. However, the thirst for knowledge depends very much upon the preference of the unit by the staff nurses. Therefore, it is vital to seek and consider the preference of the staff for employment upon graduation. If the clinical area is the preferred choice of the staff, then they will have more passion for learning. As professional nurses, they need to report assessment findings like the signs of deterioration or restlessness among patient and not just blood results. They are also required to examine the patient and report the physical examination, describe the findings, and not reporting just laboratory numbers. The professional nurses need to understand the severity of medical problems as minor or major before calling the doctors, especially at night. This would help doctors to make accurate decisions and give telephone orders prior visiting the patients. Never stop learning and think to know everything.

\section{ii. Experience and Quality of nursing care:}

The gap in knowledge can create a lack of confidence, and at times, junior nurses do not understand the instructions given to them, and they need time to get the experience. However, on the positive side, junior nurses can be easily moulded. Seven (41\%) of the doctors answered that senior nurses might not have much paper qualification, but they are very competent at their work. However, at times, senior nurses also can have attitude problems.

\section{iii. Medical English:}

Registered nurses need to use medical English for workplace communication. Five (29\%) doctors answered: "We doctors usually speak in English, but sometimes the nurses have difficulty to understand what we say." Understanding of medical terms is crucial for effective communication within the units. The nurses should be able to identify and package necessary information about the patients before case reporting to the doctors.

\section{iv. Self-confidence:}

Nurses must not feel shy or inferior. Five (29\%) doctors answered that young nurses do not ask back the doctors even if they do not understand. They lack assertiveness. When they were asked for suggestions and opinions, they do not talk. However, it is very individualistic. Doctors commented that criticism is for improvement of performance and not for finding fault.

\section{v. Ability to build rapport with patients:}

Nurses are with patients all the time and as such, they should be able to build closeness with patients. They need to Interact with patients, their parents, and build rapport to promote nursing. The essential element in building rapport is communication. Good nursepatient communication will contribute to early detection of problems and enhance nurse-doctor communication.

\section{vi. Stress management skills:}

Four $(24 \%)$ of the doctors agreed that nurses are in stress because of a double shift, attending a patient call, preparing the patient to Operation Theatre, attending 
rounds, handling dresses and importantly handling patients and their relatives with a different attitude. Stress could hinder their efficiency in performing the nursing job and minimize the time available for effective inter-professional communication.

\section{vii. Critical thinking and Clinical judgement skills:}

Two (12\%) doctors felt that nurses poorly apply their theoretical knowledge in practice. The doctors are more concerned that nurses are not using critical thinking and clinical judgment skills. If they do not identify the signs and symptoms of critical conditions and communicate accurately during the early stage itself, they may put patients at risk.

\section{viii. Perceived Power of authority:}

Some of the junior nurses feel Inferior and does not put forward suggestion or opinions regarding patient care. They should not be afraid to ask questions. Nurses must not have Inferiority complex while dealing with the specialists. However, this is highly related to the personality of both the doctors and the nurses. The age gap between junior nurses and doctors also contribute to the inferiority complex.

\section{ix. Passion to work:}

If the staff have a passion for working, they will have motivation in learning, will be dedicated to patient care and does must not complain about patients. Conversely, if the new staff is not excellent in one unit, he must be guided to find out the area of interest. The potentials in each staff must be identified. For example, some may not be good in one ward but may perform well in the Operating Room. During the student time itself, they should be able to identify their clinical area of interest.

\section{$x$. Stress and personal factors of nurses:}

The health of nurses is often neglected and more medical benefits and counselling are needed to be offered for nurses. These factors can indirectly contribute to better communication.

\section{Work environment attributes}

\section{Workload of nurses:}

Nurses are burdened with a lot of non-nursing work. Usually in the morning, they are swamped with meeting the demands of everyone in the units, both patients and healthcare providers. Heavy workload is especially true in the paediatric ward as they need to entertain the parents and all petty issues. They are also burdened with too much paperwork and sometimes double shift if there is a critical shortage of staff in the unit. Four doctors $(24 \%)$ answered that nurses were so busy in doing nonnursing works like confirm guarantee letter form of the patient on patient discharge, check hospital bill, and other such work. So, they find less time for nursing work.

\section{ii. Team work among nurses:}

Nurses must tune to the situation and learn to adjust to the ward situation. They must be flexible at work for the smooth running of the ward and safety of the patient. The unit manager must bring out nurses' issues to be discussed during ward meeting. Nursing care promotes hospital and teamwork will enhance the quality of care provided and thus promote the hospital. The respondents also suggested tagging of junior nurses with the seniors to inculcate the ward culture. Seniors need to teach new staff or students.

\section{iii. Preceptorship/Mentoring:}

The senior preceptors must guide the juniors and help them develop self-confidence. A good preceptor can be of immense help to the junior staff nurses in shadowing and developing good communication skills.

\section{iv. Appreciation of nurses by the organization:}

Doctors also suggest that nurses upon graduation should be given preferred discipline to work and advise the nurses to be committed more on their work. If nurses are happy, they will volunteer to give quality patient care. We cannot force people to work. Being happy generates motivation and interest, and all the elements help each other for quality patient care.

\section{Suggestions by Doctors:}

The following are the suggestions given by the doctors in improving the doctor nurse communication.

Table 2: Recommendations for minimizing communication gap by doctors

\begin{tabular}{|l|l|}
\hline No. & Suggestions \\
\hline 1. & $\begin{array}{l}\text { Nurses need to equip themselves with sound knowledge in the related } \\
\text { discipline. }\end{array}$ \\
\hline 2. & Improve Physical examination skills relevant to each discipline. \\
\hline 3. & $\begin{array}{l}\text { Nurses need to improve medical English language proficiency and } \\
\text { converse using medical English. Use medical terminologies in both } \\
\text { verbal and written communication. Professionals must } \\
\text { communication among themselves using professional language. }\end{array}$ \\
\hline 4. & $\begin{array}{l}\text { Management should o ffer preferred choice of unit to new staff to } \\
\text { motivate them to do their best. }\end{array}$ \\
\hline 5 & $\begin{array}{l}\text { New graduate nurses need structured preceptor system to adapt to the } \\
\text { hospital environment and unit culture. }\end{array}$ \\
\hline 6. & $\begin{array}{l}\text { Hospitals must appreciate nurse's contribution and motivate them. } \\
\text { Nurses' own health needs must be a cknowledged and counsel led } \\
\text { appropriately. Need to pay more attention to their welfare needs. }\end{array}$ \\
\hline 7. & Improve hospital system to minimize non nursing work of nurses. \\
\hline 8. & \begin{tabular}{l} 
Promote activities to promote team spirit among nurses in all units. \\
\hline
\end{tabular}
\end{tabular}




\section{DISCUSSION}

Effective communication is critical during interactions that occur among healthcare staff and patients every day. If effective communication is absent, the quality of service rendered to patients as well as staff is compromised. Communication competence is, therefore, one of the essentials to be mastered by medical professionals. The purpose of the study was to determine the factors contributing to the communication gap between nurses and doctors. The study findings revealed three categories of determinants related to the communication gap between doctors and registered nurses. The factors contributing to the communication gap among doctors and nurses are multifactorial. The factors may directly or indirectly contribute to the communicationgap.

The first category was related to nursing education. The factors which were highlighted to be a hindrance to communication competency of nurses in the undergraduate training were lack of aptitude testing during the selection process, ineffective nursing training, and inadequate communication skill competency assessment procedures.

The respondents highlighted the importance of reviewing the entry criteria's like aptitude screening as part of recruitment for improving the credentials of students enrolled into the nurses training. The assumption is that a high aptitude test score may result in producing nursing graduates with higher nursing qualities. However, one of the studies has shown that the aptitude test which determines the intelligence of the individuals may not have any association with nursing care qualities. A study among 227 second-year nursing students aimed to identify the relationship among the score of aptitude test, achievement, and qualities of nursing students. The findings suggested that the nursing traits like caring, compassion, commitment, and connections are not correlated with the aptitude score and achievement score of the nursing students. The study findings concluded that effective nursing qualities are independent of the cognitive ability of nursing students in the aptitude and achievement tests (Bello, 2017).

Obtaining a high score in aptitude test does not guarantee practical nursing care qualities (Magno, 2010). In another study among 95 high schools, counselors commented that kindness, compassion, and obedience are the critical qualities needed for the nursing profession. The respondents rated leadership, assertiveness, and decision-making skill as the least necessary qualities for a nurse. As inference, very few studies have explored the relationship between aptitude score of student nurses and nursing care qualities. Thus more research is required to identify if the aptitude test is essential for admission into nursing studies (Blasdell \& Hudgins-Brewer, 1999). Till date, no studies have been conducted to identify if the aptitude test score is associated with the communication skill of Registered nurses.

Another viewpoint highlighted by the doctors was the need for improving the standards of undergraduate nurses training with more structured clinical practice and detailed competency assessment, including assessment of communication skills. In the local context of this study, the communication competencies are examined as part of Objective Structured Clinical Examination (OSCE) and as well as during clinical assessment in each semester. However, communication skill as a separate core competency is not being assessed.

In another study, the researchers investigated how theoretical and practical elements of communication is taught in the undergraduate nursing curriculum. The findings concluded that the communication module did not have a clear goal and assessment. The practical session included role-playing video recording and reflective learning. In the local setting of the study, the revised curriculum for undergraduate nursing students has included communication and counseling as a separate module taught by registered counselors (Wikström \& Svidén, 2011).

The second category includes graduate nurse attributes. Knowledge, communication, courage, and confidence are highly associated with this. Knowledge is an essential element for professional like nurses in determining the self-confidence. Self-confidence will boost the courage to speak up. All the 15 respondents commented the need for sound knowledge in the specialty area as the foundation for practical communication skills among doctors and nurses. However, till date, no study had been conducted to associate nurse's knowledge and communication skills.

The study also emphasized the lack of language fluency as one of the reasons for poor communication skills between nurses and doctors. Medical jargons in the English language are used by medical professionals to record and explain specific information on treatment procedures to patients. In order to provide professional 
service appropriately, medical professionals need to be competent in their listening, speaking, and writing skills to interact with patients and to respond to their inquiries. Medical English cannot be taught at this level in the same methods of primary English language teaching. Careerspecific, highly technical language must be contextually based, and the aim of learning English for medical professionals is not to learn grammar and structure primarily but to acquire and use the language of practice as well as for social relations within the career. In the Malaysian context, hospitals need to accommodate the demands of patients of all walks of life, foreign doctors, and specialists trained in other countries. Therefore medical English is essential in medical communication (Perinpasingam et al., 2015).

Bello (2017) also stated the personality of nurses as another cause for poor communication. A poor selfimage, possibly combined with negative self-talk, can set the stage for ineffective interpersonal communication. This is especially true for junior nurses. The study findings were similar to the findings of this study in which powerlessness or inferiority complex by nurses was identified to be one of the contributing factors for poor communication among doctors and nurses. Nurses tend to adopt a servant role, paternalistic, or collegian role with the physicians.

The third category of factors contributing to poor nurse-doctor communication as perceived by doctors includes working environment-related factors. A working environment which offers motivations, trust, communication, respect, personal and team support, and allows independence is significant in minimizing the prevalence of burnout syndrome among nurses. An equally important factor for effective collaboration is the personality of health professionals involving both self-confidence and emotional maturity (Ubani, 2015). The work environment factors identified in this study were the high workload of nurses, teamwork among nurses, preceptorship, and appreciation of nurses by the organization. Bello (2017) in the systematic review exclaimed that the work environment factors affecting communication skill among nurses as high workload of nurses, lack of sufficient time, and lack of privacy. The findings are similar to these study findings in which high workload was one of the major factors contributed to nurse-physician communication gap. Many specialists doing rounds at the same time, answering calls from patients and relatives, responding to telephone, a heap of paperwork and electronic recording contributes to the heavy workload of the nurses. Nurses, in general, are more involved with non-nursing work, which increased their burden and indirectly contributed to poor communication skills.

Effective preceptorship can help to boost the confidence of junior nurses. Effective communication skills are learned through preceptorship by role modeling (Puttagunta \& Pulipati, 2017). One of the respondents highlighted that junior nurses are very easy to mold. Hence a preceptor with excellent preceptor skills can use role model techniques of good clinical communication, and the preceptee will benefit greatly through the preceptor influence. The doctors recommended to tag junior nurses with seniors and slowly learned to develop self-confidence.

Effective team working has become a core concern for most organization. Teamwork is essential in effective communication. Many factors influenced the team's performance like team members' diversity and the roles they play in the team. Thus, the team formation is identified as a critical factor that influences team performance (Burke, Boal \& Mitchell, 2004).

A research was conducted on SBAR (Situation, Background, Assessment, and Recommendation) communication techniques to improve communication skills among nurse-physician. The researcher collected baseline data from the nurses and physician within the hospital. From the baseline data, the result indicated that most healthcare providers admit the gaps in the communication and the necessity for the improvements. Based on the survey conducted, the SBAR technique was introduced in the hospital to standardize the transfer of information to nursing staff and physicians. The researcher has identified few elements for the training such as flyers, pocket cards, and reference guide for the nurses.

Additionally, the researcher also created laminated nursing shift report templates and reported to physician checklists for all inpatient nursing services over telephone. After one year of implementation, the same hospital was re-evaluated on the efficacy of the SBAR technique. There were $20 \%$ to $40 \%$ of improvement on the SBAR technique during shift report. Improvement of communication skill was observed among the novice and experienced nurses while making phone calls to the physician during a critical situation (Toghian Chaharsoughi,Ahrari \& Alikhah, 2014). 


\section{Limitations}

Several limitations to the proposed studies have been identified. The first respondents were not assigned randomly. A purposive sampling has been recruited and it may contribute to confounding factors which may influence the results. Second limitation was the time constraint. The data collection involved travelling to various hospitals and collecting data as per the convenience of the respondents. Moreover, the study findings involve perception by doctors in a specific healthcare organization and may not be generalizable to other healthcare settings.

\section{RECOMMENDATIONS}

This study employed a descriptive qualitative method. The recommendation is to use Delphi technique which may assist to refine and generalize the findings of the study.

\section{CONCLUSION}

Effective clinical communication is respectful, clear, direct, and explicit. Conversely, deficient communication can create stress, frustration, distrust and leads into inferior care and increased risk of medical errors. The nurses and doctors have a vital role in creating good relationship to ensure superior quality of care for the patients. The study showed that communication skills were affected by the undergraduate nursing training, personal attributes of nurses and working environment factors. From the findings of the study, the researcher concludes that there is a need to address the factors highlighted to improve nurse-doctor communication skills. The findings may be helpful for the nursing schools to identify the gaps in communication skill development of nurses and adopt necessary steps in improving the communication skill of nurses of the newly graduated nurses.

\section{REFERENCES}

Bello, O. (2017). Effective Communication in Nursing Practice: A literature review. Bachelor's Thesis Degree Programme in Nursing 2017 (5), pp 6. Retrieved from: http://www.theseus.fi/bitstream/handle/10024/ 130552/OPE THESIS Final submitted.pdf?sequence $=1$.

Blasdell, A. \& Hudgins-Brewer, S. (1999). High school counselors' perceptions of the academic and personality attributes important for a career in nursing. Journal of Nursing Education, 38(4), pp 176-179.

Burke, B.M., Boal, J. \& Mitchell, R. (2004). Communicating for better care: improving nurse-physician communication. American Journal of Nursing, 104(12), pp 47-48.

Hsu, L.L., Chang, W.H. \& Hsieh, S.I. (2015). The Effects of Scenario-Based Simulation Course Training on Nurses' Communication Competence and Self-Efficacy: A Randomized Controlled Trial. Journal of Professional Nursing, 31(1), pp 37-49.

Magno, C. (2010). Integrating Nursing Quality with Achievement and Aptitude: Towards Assessing Nursing Potential. International Journal of Research and Review, 4(March), pp 37-49.

Perinpasingam, P.T.S., Arumugam, N., Thayalan, X. \& Maniam, M. (2015). Needs Analysis on the Importance of English Language Skills for Workplace: Trainee Architects. International Review of Basic and Applied Sciences, 3(10), pp 129-137.

Puttagunta, S.B. \& Pulipati, S. (2017). Effective precepting to enhance communication skills of pharmacy students. Pharma Times, 49(7), pp 9-13.

Sorra, J., Gray, L., Streagle, S., Famolaro, T., Yount, N. \& Behm, J. (2016). AHRQ Hospital Survey on Patient Safety Culture: User's Guide. (Prepared by Westat, under Contract No. HHSA290201300003C). AHRQ Publication No. 15-0049-EF. Rockville, MD: Agency for Healthcare Research and Quality. Retrieved from: https://www. ahrq.gov/sites/default/files/wysiwyg/professionals/quality-patient-safety/patientsafetyculture/hospital/ userguide /hospcult.pdf 
Stein, L.I. (1967). The Doctor-Nurse Game. Archives of General Psychiatry, 16(6), pp 699-703.

Toghian Chaharsoughi, N., Ahrari, S. \& Alikhah, S. (2014). Comparison the Effect of Teaching of SBAR Technique with Role Play and Lecturing on Communication Skill of Nurses. Journal of Caring Sciences, 3(2), pp 141-147.

Ubani, T.O. (2015). Nurse-Physician Communication Tools to Enhance use of Nursing Evidence-Based Protocols. Retrieved from: http://search.ebscohost.com/login.aspx?direct $=$ true \&db=ccm\&AN=109786594\&site $=$ ehostlive \&scope $=$ site.

Wikström, B.-M. \& Svidén, G. (2011). Exploring communication skills training in undergraduate nurse education by means of a curriculum. Nursing Reports, 1(1), e 7. 